\title{
Evaluación de la intervención "Malnutrición por exceso de peso en la primera infancia" con base en el modelo de teoría del cambio, Medellín, Colombia, 2017
}

\author{
Evaluation of the "Early Childhood Malnutrition due to Overweight" Intervention \\ Based on the Theory of Change Model, Medellín, Colombia, 2017 \\ Avaliação da intervenção "Má nutrição por excesso de peso na primeira infância" \\ baseada no modelo de teoria da mudança, Medellín, Colômbia, 2017
}

Cristina María Mejía Merino"; Camilo Noreña Herrera²; Santiago Antonio Flórez Vanegas³; Alejandra Marín Uribe; Verónica María Lopera Velásquez ${ }^{5}$

1 Nutricionista, magíster en Salud Pública, Universidad de Antioquia. Colombia. cristinameja@gmail.com - oRciD: https://orcid.org/00000003-2782-9567

2 Administrador en Salud, magíster en Ciencias de la Salud, Universidad de Antioquia. Colombia. camilo.norena@udea.edu.co. ORCID: https://orcid.org/0000-0002-5671-8463

3 Profesional en Sistemas de Información en Salud, especialista en Estadística. Universidad de Antioquia. Colombia. santiago.florex@ gmail.com. ORCID: https://orcid.org/0000-0003-3025-0177

4 Odontóloga, magíster en Salud Pública. Universidad de Antioquia. Colombia, alejandra.marinu@udea.edu.co https://orcid.org/00000003-1302-9075

5 Bacterióloga, magíster en Gobierno y Políticas Públicas, Alcaldía de Medellín. Colombia. veronica.lopera@medellin.gov.co. ORCID: https://orcid.org/0000-0002-5189-5946

Recibido: 30/11/2018. Aprobado: 18/11/2020. Publicado: 27/01/2021

Mejía-Merino CM, Noreña-Herrera C, Flórez-Vanegas SA, Marín-Uribe AL; Lopera-Velásquez. VM Evaluación de la intervención "Malnutrición por exceso de peso en la primera infancia" con base en el modelo de teoría del cambio, Medellín, Colombia, 2017. Rev. Fac. Nac. Salud Pública. 2021;39(1):e340565. Dor: https://doi.org/10.17533/udea.rfnsp.e340565

\section{Resumen}

Objetivo: Evaluar una intervención de base poblacional denominada "Malnutrición por exceso de peso en la primera infancia", implementada con recursos del programa de Presupuesto Participativo en Salud de Medellín, Colombia, en 2017. Método: Perspectiva de investigación de tipo evaluativa, con base en el modelo de teoría del cambio, utilizado en el campo de la evaluación de programas y proyectos en salud. Se desarrolló un estudio de caso de método mixto convergente e igualdad de estatus. El caso de análisis fue la implementación y los resultados de la intervención "Malnutrición por exceso de peso en la primera infancia". La evaluación se dio en dos fases: 1) la planeación de la evaluación, y 2) la evaluación de los procesos durante su implementación y de los efectos al finalizar la intervención. Resultados: Al finalizar la

* Artículo resultado del proyecto: "Evaluación de los procesos de priorización, implementación y de los efectos en la población de los componentes del programa de Presupuesto Participativo en Salud del municipio de Medellín". Convenio interadministrativo 4600071225 de 2017, entre la Alcaldía de Medellín y la Universidad de Antioquia. 
intervención, no se observaron efectos significativos para los indicadores antropométricos de los niños y las niñas. En el peso para la talla, la mediana al iniciar el tratamiento era de 2,52, y al finalizarlo, de 2,49 $(p(0,174)>0,05)$. Para el índice de masa corporal, la mediana al iniciar el tratamiento era de $2,4$, y al finalizarlo, de 2,3 ( $p(0,236)>0,05)$. Sin embargo, de forma individual hubo variaciones en las desviaciones estándar de los valores $\mathrm{Z}$ antropométricos de los niños y las niñas; esto se explica porque en la evaluación de los procesos se identificó el compromiso, la aceptabilidad y la adopción de las recomendaciones nutricionales y psicosociales por parte de los padres y cuidadores. También se encontraron cambios no previstos en el modelo de teoría del cambio, los cuales hacen referencia a variaciones antropométricas en los cuidadores de los niños y las niñas. Conclusión: Los efectos individuales a corto plazo que se dieron en la intervención "Malnutrición por exceso de peso en la primera infancia" favorecieron variaciones antropométricas y el mejoramiento del estado nutricional de niños, niñas y cuidadores, lo cual se explica por la adopción de estilos de vida saludables, lo que permitió alcanzar el cambio global definido para la intervención en el modelo de teoría del cambio.

-Palabras clave: Malnutrición, salud del niño, evaluación de programas y proyectos de salud, participación de la comunidad, Colombia

\section{Abstract}

Objective: To evaluate a population-based intervention named "Early Childhood Malnutrition due to Overweight" implemented with resources of the Participatory Budget for Health program of Medellín, Colombia, in 2017. Methodology: Evaluative research perspective, based on the theory of change model, used in the field of health projects and programs evaluation. A case study of the convergent mixed method and equality of status was conducted. The case under analysis was the implementation and the results of the intervention "Early Childhood Malnutrition due to Overweight". The evaluation was made in two stages: 1) evaluation planning, and 2) evaluation of the processes during their implementation and the effects at the end of the intervention. Results: At the end of the intervention, significant effects in the anthropometric index of the children were not observed. The median weight-for-height at the beginning of the treatment was 2.52; at the end it was 2.49 (p (0.174) > $0.05)$. The median body mass index at the beginning of the treatment was 2.4 , and at the end, it was $(p(0.236)>0.05)$.
Nevertheless, there were individual variations in the standard deviations of the anthropometric Z-score of children, which is explained due to commitment, acceptability, and adoption of the nutritional and psychosocial recommendations by parents and child caregivers, which were identified during the evaluation of the processes. Changes not expected according to the theory of change model were also found. Those changes refer to the anthropometric variations in the child caregivers. Conclusion: The short-term individual effects that occurred during the "Early Childhood Malnutrition due to Overweight" intervention contributed to the anthropometric variations and the nutritional status improvement of the children and child caregivers. This is explained by the adoption of healthy lifestyles, which allow to reach the global change established for the intervention in the theory of change model.

-Keywords: malnutrition, child health, evaluation of health projects and programs, community participation, Colombia.

\section{Resumo}

Objetivo: Avaliar uma intervenção de base populacional denominada "Má nutrição por excesso de peso na primeira infância" implantada com recursos do programa de Orçamento Participativo em Saúde de Medellín, Colômbia, em 2017. Metodologia: Perspectiva de estudo do tipo avaliativo, com base no modelo de teoria da mudança, utilizado no campo da avaliação de programas e projetos em saúde. Foi desenvolvido um estudo de caso de método misto convergente e igualdade de status. $\mathrm{O}$ caso analisado foi a implantação e os resultados da intervenção "Má nutrição por excesso de peso na primeira infância”. A avaliação foi feita em duas etapas: 1) O planejamento da avaliação, e 2) A avaliação dos processos durante sua implantação e dos efeitos ao terminar tal intervenção. Resultados: Ao concluir a intervenção, não se observaram efeitos significativos para os indicadores antropométricos das crianças. No quesito para o tamanho, a média ao iniciar o tratamento era de 2,52 e ao finalizá-lo, de 2,49 (p $(0,174)>0,05)$. Para o índice de massa corporal a média ao iniciar o tratamento era de 2,4 e ao finalizá-lo, de $2,3(\mathrm{p}(0,236)>0,05)$. Porém, de maneira individual houve variações nos desvios padrão dos valores $\mathrm{Z}$ antropométricos das crianças; isto se explica porque na avaliação dos processos foi identificado o compromisso, a aceitação e a adoção das recomendações nutricionais e psicossociais por parte dos pais e cuidadores. Também foram encontradas mudanças não 
previstas no modelo de teoria da mudança, as quais se referem a variações antropométricas nos cuidadores das crianças. Conclusões: Os efeitos individuais em curto prazo que se deram na intervenção "Má nutrição por excesso de peso na primeira infância" foram favoráveis às variações antropométricas e ao melhoramento do estado nutricional das crianças e cuidadores, o qual se explica pela implantação de estilos de vida saudáveis, o que permitiu alcançar a mudança global definida para a intervenção no modelo de teoria da mudança.

--Palavras-chave: Má nutrição; saúde infantil; avaliação de programas e projetos de saúde; participação da comunidade, Colômbia.

\section{Introducción}

Para la Organización Mundial de la Salud (OMs), el sobrepeso y la obesidad constituyen un problema global de salud pública, con una tendencia en aumento en las últimas cuatro décadas. Este problema no es ajeno en la infancia, evidenciándose el incremento en los porcentajes de obesidad infantil y adolescente, "que eran menores que $1 \%$ en 1975 , equivalente a 5,5 millones de niños y niñas, hasta llegar a $8 \%$ en 2016 , aproximadamente 74 millones de niños y niñas en el ámbito mundial" [1].

Investigadores como Urrutia reportan que, en América Latina, 4 millones de niños y niñas menores de 5 años padecen sobrepeso u obesidad [2]. Para la OMS, si las tendencias de 2017 se mantienen, los niveles mundiales de obesidad en la población infantil y adolescente superarán en 2022 los de insuficiencia moderada o grave (peso inferior para la edad) en el mismo grupo de edad [1].

De acuerdo con Hurtado et al., "En Colombia, en la última década el exceso de peso ha venido aumentando en todos los grupos de edad. Según cifras reportadas por dos Encuestas Nacionales de Situación Nutricional, la obesidad en los niños de 0 a 4 años pasó de 3,1\% a 5,2\% entre 2005 y 2010" [3, p. 67].

En Medellín, en 2015, para los niños y las niñas de 0 a 4 años, el riesgo de presentar obesidad fue del $7 \%$ [4]. Según el "Plan de seguridad alimentaria y nutricional del municipio de Medellín 2016-2028" [5], la prevalencia de obesidad ( $5 \%$ ) fue más alta en niños y niñas menores de 2 años $(6,6 \%)$, y en relación con el sobrepeso en menores de 5 años o primera infancia, la prevalencia en la zona urbana $(15 \%)$ fue menor que en la rural $(20 \%)$, según el indicador antropométrico peso para la talla $(\mathrm{P} / \mathrm{T})$.

\section{Respuestas gubernamentales y sociales implementadas y la importancia de su evaluación: el caso de "Malnutrición por exceso de peso en la primera infancia"}

El problema de sobrepeso y obesidad infantil genera la necesidad de desarrollar intervenciones para prevenir esta condición a lo largo de la vida de la persona, considerando las complicaciones en el tiempo, en caso de ausencia de intervenciones [6,7]. Una respuesta en Medellín fue la intervención "Malnutrición por exceso de peso en la primera infancia”, implementada en 2017 con recursos del programa de Presupuesto Participativo en Salud (PP) del periodo de gobierno 2016-2019, donde se destinó el 5\% de los recursos del Plan de Desarrollo Municipal a los problemas priorizados por las comunidades [8].

La intervención "Malnutrición por exceso de peso en la primera infancia" fue realizado con niños y niñas en primera infancia, diagnosticados con sobrepeso $\mathrm{u}$ obesidad, según el indicador $\mathrm{P} / \mathrm{T}$ y el índice de masa corporal para la edad (IMC/E). Incluyó acciones en nutrición y alimentación, encaminadas a favorecer un adecuado estado nutricional de esta población, y acciones de información, educación, comunicación y movilización, para fomentar estilos de vida saludable. Estas acciones se llevaron a cabo en las comunas 5, Castilla, y 14, El Poblado, del municipio de Medellín.

Asimismo, la intervención contó con un equipo interdisciplinario (nutricionistas, médicos, pedagogos y profesionales del área psicosocial), quienes realizaron actividades de valoración nutricional inicial al niño o la niña atendidos, y seguimientos grupales. Esto último incluyó: valoración médica, nutricional y actividades pedagógicas, dos visitas de seguimiento nutricional, y una visita al grupo familiar por parte de un profesional psicosocial.

Además, se tenía un equipo interdisciplinario para el análisis de casos especiales y la definición de una actividad comunitaria colectiva denominada "Salud a nuestro barrio". En la Figura 1 se identifican las actividades programadas y ejecutadas durante la intervención.

La importancia de evaluar programas e intervenciones en salud pública de base poblacional como "Malnutrición por exceso de peso en la primera infancia" radica en:

1. La preocupación por el costo-efectividad [9], dados los costos de su implementación y los beneficios obtenidos en la salud de la población.

2. Lograr aprendizajes institucionales que permitan el entendimiento continuo y sistemático de los efectos conseguidos por las acciones implementadas, tomando en cuenta los objetivos fijados inicialmente.

3. Asegurar su sostenibilidad en el tiempo. 
4. Mayor exigibilidad de los ciudadanos y líderes comunitarios por conocer los resultados de los programas implementados en la población $[9,10]$.

5. Las implicaciones de liderazgo, compromiso, credibilidad y transparencia que deben ejercer los gobiernos locales, en este caso, la autoridad sanitaria muni- cipal (Secretaría de Salud), frente a los recursos que hacen parte del programa de Presupuesto Participativo en Salud de Medellín, donde son las comunidades las que eligen sobre qué problemas o necesidades invertirlos.

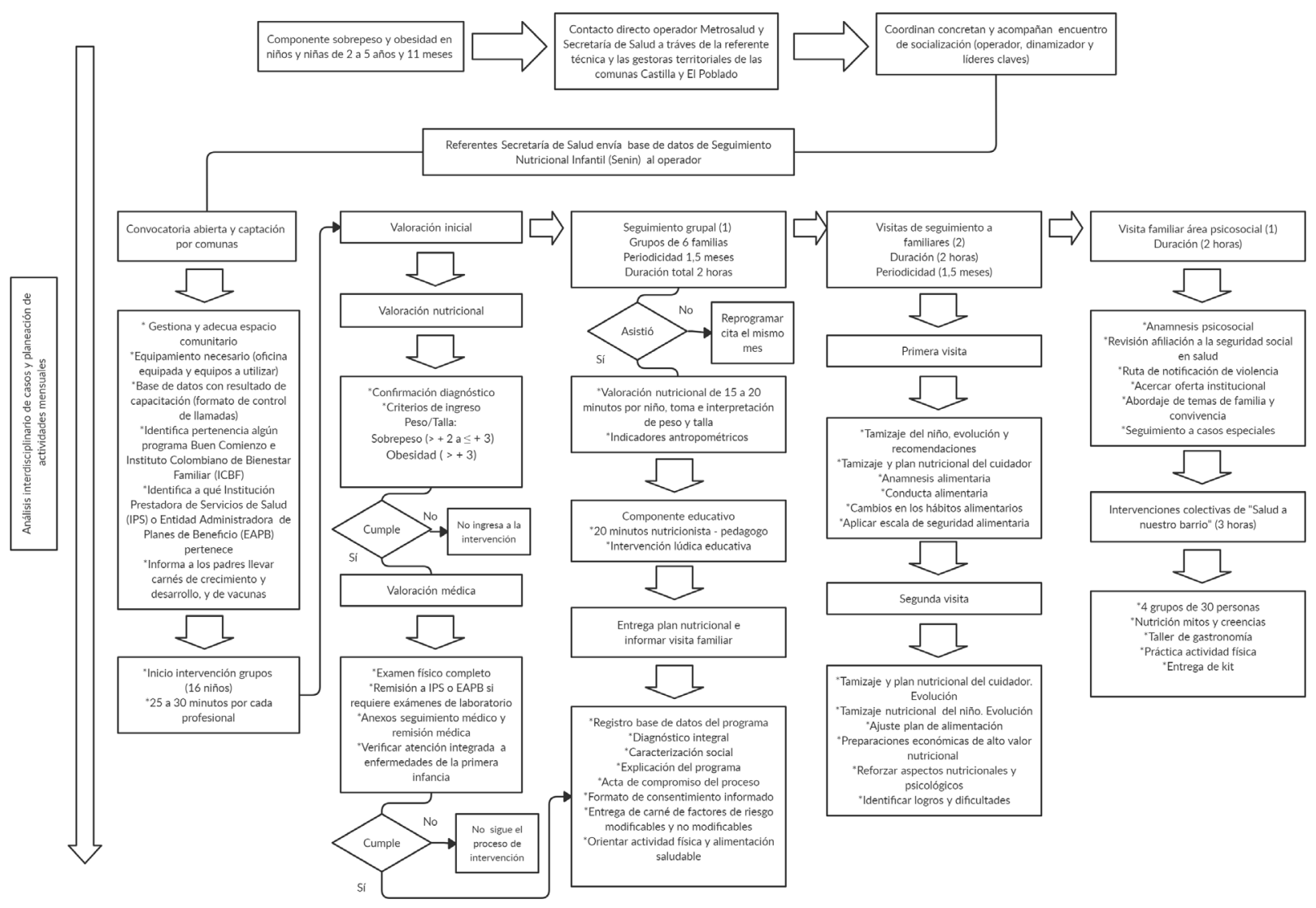

Figura 1. Flujograma de la intervención "Malnutrición por exceso de peso en la primera infancia" del programa de Presupuesto Participativo en Salud de Medellín, Colombia, en 2017

De acuerdo con lo anterior, el equipo evaluador utilizó el modelo de teoría de cambio, insumo fundamental para la evaluación de programas y políticas $[9,11,12]$. De este modo, el objetivo del presente estudio fue evaluar una intervención de base poblacional denominada "Malnutrición por exceso de peso: sobrepeso y obesidad en la primera infancia", implementada con recursos del programa de Presupuesto Participativo en Salud de Medellín, Colombia, en 2017. La importancia de la evaluación de esta estrategia radicó en el interés de la Secretaría de Salud de Medellín y de las comunidades que lo priorizaron con los recursos de presupuesto participativo, en conocer los resultados de esta intervención en la salud de las niñas y los niños que se encontraban con esta condición de sobrepeso y obesidad, dada la necesidad de contar con evidencia científica para el mejoramiento continuo de la intervención, además de tomar decisiones sobre su continuidad en el tiempo, considerando los resultados encontrados.

\section{Metodología}

Investigación de tipo evaluativa de la intervención "Malnutrición por exceso de peso en la primera infancia". La evaluación se hizo con base en el modelo de teoría de cambio de la intervención. Se desarrolló un estudio de caso mediante un método mixto convergente con igualdad de estatus $[13,14]$. 
El modelo de teoría de cambio se denomina de diferentes formas según el área disciplinar; por ejemplo, "ruta de cambio" (pathway of change) o "teoría de acción" (theory of action) [11,15]. Pero independiente de esto, una definición que recoge su sentido es:

[...] un amplio análisis de una situación que requiere modificarse a fin de alcanzar un cambio positivo. Este enfoque de comprensión e intervención en la realidad parte de una visión de éxito (cambio de largo plazo, imagen objetivo, o macro cambio) e identifica un conjunto de resultados primarios, secundarios, terciarios (todos ellos con precondiciones "unos de otros") que articuladamente permite alcanzar el cambio de largo plazo deseado. Tales precondiciones (resultados a diferente nivel) constituyen los elementos necesarios y suficientes para alcanzar el cambio planteado en concordancia con un conjunto de supuestos relacionados [12].

Igualmente, esta evaluación incorporó el enfoque de evaluación centrada en la utilización [16,17], en clave de fortalecer o reorientar los proyectos de PLyPP en salud y para llevar a cabo los acompañamientos a las comunidades, operadores y demás actores participantes en los procesos de implementación $[6,18]$.

Desde la perspectiva de Ridde et al., la evaluación se concibe como "una serie de cuatro fases interdependientes" [14, p. 23], que incluye: 1) la planificación de la evaluación, 2) la implementación de la evaluación, 3) un momento evaluativo/reflexivo de la evaluación, y 4) la sostenibilidad del proceso de evaluación a largo del desarrollo de la intervención o la política. Para efectos de esta evaluación, sólo implementamos las fases 1 y 2 .

\section{Planeación del alcance de la evaluación}

La fase de "planeación" de la evaluación implicó una revisión documental de la intervención "Malnutrición por exceso de peso en la primera infancia" para identificar su modelo de teoría del cambio, lo cual permitió identificar y delimitar los siguientes elementos de la intervención:

1. Problema o necesidad a resolver.

2. Intervenciones a desarrollar para resolver el problema.

3. Características de la intervención y de sus actividades.

4. El proceso de implementación definido.

5. Las circunstancias externas consideradas.

6. Las características de la población participante.

7. Resultados esperados a corto plazo.

8. Resultados a mediano plazo.

9. Resultados a largo plazo en caso de mantenerse durante 5 años la intervención.

Este momento de definición del modelo de cambio es la primera etapa y la crucial en todo el proceso de evaluación; además, permite reflexionar, con las diferentes partes interesadas, los pormenores de un programa respecto a una multitud de objetivos y alcances, los cuales incluso pueden ser replanteados [19].
Durante este proceso de planeación y delimitación del alcance de la evaluación, basados en una detallada revisión documental acerca de la intervención, se construyó un primer modelo de cambio por parte de los investigadores, que se validó y rediseñó con los actores de la Secretaría de Salud municipal y de la entidad que implementó el proyecto. Lo anterior, mediante un taller de tres sesiones, con el cual se obtuvo una segunda versión del modelo de teoría del cambio, el cual permitió delimitar el alcance de la intervención con respecto a lo que inicialmente se había establecido en la planeación de implementación de la misma.

De este modo, en la segunda versión del modelo de teoría de cambio se definió el cambio global esperado una vez finalizará la implementación de la intervención, el cual fue definido como: variaciones por encima o por debajo del indicador antropométrico $\mathrm{P} / \mathrm{T}$ (peso para la talla) en niños y niñas menores de 5 años, y el IMC/TALLA (índice de masa corporal para la talla) para los niños y las niñas mayores de 5 años.

Como desenlaces secundarios se estableció que los padres, las madres o cuidadores, y los niños y las niñas lograran sensibilizarse, reconocer e identificar los factores de riesgo asociados al sobrepeso y la obesidad. Dentro de los supuestos, es decir, elementos necesarios para lograr el cambio, se estableció: 1) contar con un equipo interdisciplinario, 2) lograr la adherencia de los participantes al proyecto, 3 ) incluir profesionales cualificados en el manejo de la malnutrición por exceso, y 4) establecer la articulación efectiva entre los líderes, el operador y la Secretaría de Salud.

\section{Implementación de la evaluación de los procesos y de los efectos de la intervención}

Es importante aclarar que la evaluación de los procesos se desarrolló de manera paralela a la implementación de la intervención [13], y los efectos al finalizar la intervención [20].

La intervención "Malnutrición por exceso de peso: sobrepeso y obesidad en la primera infancia" se implementó de acuerdo con el procedimiento de los proyectos que se ejecutan con recursos del presupuesto participativo, que incluyen cuatro momentos: 1) la socialización de la intervención ante los líderes comunitarios de las comunas 5, Castilla, y la 14, El Poblado; 2) la convocatoria para la selección de los niños y las niñas participantes de la intervención; 3) la selección de beneficiarios después de la verificación de los criterios de inclusión definidos por la intervención, y 4) el desarrollo de las diferentes actividades de salud y nutrición definidas para el logro de los objetivos y el cambio global previsto.

El desarrollo de estos momentos y procedimientos permite: 1) validar la coherencia de la intervención con la necesidad priorizada por la comunidad, 2) generar interés y expectativa en los líderes comunitarios para la 
divulgación con los habitantes de las comunas, 3) promover la participación de los habitantes de las comunas y los corregimientos, y 4) seleccionar uno o varios líderes comunitarios que acompañaran y apoyaran la implementación de los proyectos [9].

La evaluación de los procesos y de los efectos de la intervención se realizó en las dos comunas mencionadas. Participaron, en la evaluación, actores institucionales, gubernamentales, comunitarios y beneficiarios, entre ellos: 1) líderes comunitarios; 2) padres, cuidadores y el grupo familiar; 3) los profesionales de la salud que implementaron la intervención; 4) niños y niñas; 5) profesionales de la Secretaría de Salud; 5) interventores externos y 6) la coordinadora de la intervención "Malnutrición por exceso de peso en niños y niñas".

El muestreo, en el componente cualitativo del proyecto, se fue configurando durante el proceso de recolección de información, con base en el criterio de saturación de las categorías a priori, las cuales fueron: 1) los actores, 2) el marco institucional, 3) las racionalidades de los actores, $\left.{ }^{*} 4\right)$ las prácticas y tensiones, y 5) las categorías emergentes.

Paralelo al proceso de implementación de la intervención, los evaluadores desarrollaron ejercicios de participación observante, entrevistas semiestructuradas, grupos de discusión y talleres con los niños y las niñas, para la realización de dibujos, los cuales fueron apoyados con sus propios relatos.

Para la evaluación de los efectos, específicamente en el corto plazo, se llevó a cabo un diseño epidemiológico observacional longitudinal, con el cual se analizaron las dos mediciones desarrolladas a los niños, las niñas y las personas adultas participantes, al inicio y al final de la intervención.

La población de estudio para el análisis cuantitativo estuvo conformada por 172 niños y niñas, entre los 2 y 5 años y 11 meses, con riesgo de sobrepeso o diagnóstico de sobrepeso u obesidad. De estos, 14 abandonaron el programa, quedando una población de 158 niños y niñas, de los cuales solo 48 participaron de todas las actividades desarrolladas en el programa y contaban con mediciones al inicio y al final (lo anterior, aunque se reporta para efectos de la metodología, es un aspecto que se retoma en los resultados, y que da cuenta de la adherencia de la población al programa, que fue uno de los supuestos necesarios para lograr los cambios definidos en el modelo de cambio).

De este modo, con estos 48 niños y niñas profundizamos el análisis de la intervención y se evaluaron los efectos de la intervención mediante indicadores antro-

* Las racionalidades hacen referencia a las posiciones que asumen las personas sobre un tema o un asunto, dada su experiencia con respecto al asunto en cuestión. Las enmarcamos en: 1) racionalidades políticas; 2) racionalidades técnicas, y 3) racionalidades sobre las necesidades de la población. pométricos. Los participantes se diferenciaron de acuerdo con las actividades recibidas, a fin de determinar las variaciones en el $\mathrm{P} / \mathrm{T}$ de los niños y las niñas menores de 5 años, y del IMC de los mayores de 5 años.

Las mediciones al inicio y al final fueron tomadas por la institución operadora que implementó el programa. Esta información fue utilizada para el análisis de la evaluación de efectos por parte de los investigadores y autores del presente artículo, es decir, constituyó un análisis de fuentes secundarias.

\section{Análisis de la información}

El análisis de la información cualitativa se desarrolló mediante análisis formal de contenido; se procedió a realizar codificación abierta y construcción de una matriz categorial, con base en un modelo analítico para estudiar: 1) cambios en los actores participantes, especialmente niños y niñas, y sus cuidadores; 2) el desarrollo del marco institucional de la intervención durante su implementación; 3) las racionalidades de los múltiples actores, y 4) las prácticas y tensiones de los actores durante la implementación.

Por su parte, para los datos cuantitativos se realizaron análisis descriptivos de variables antropométricas, socioeconómicas y alimentarias, apoyados por un análisis de correspondencias múltiples. Se efectuó un análisis de supervivencia, para evaluar la adherencia de los participantes a las actividades, y un análisis de comparación de muestras pareadas (Prueba de U de MannWhitney) de la población de estudio al inicio y al final de la intervención, con el fin de determinar las diferencias en sus mediciones antropométricas, a través de las desviaciones estándar de los valores $\mathrm{Z}$ antropométricos (Puntaje Z) [21], lo cual permitió, a su vez, identificar las variaciones en los indicadores antropométricos $\mathrm{P} / \mathrm{T}$ e IMC. Igualmente, se analizaron los datos de los cuidadores que registraron seguimientos antropométricos para el indicador IMC. Además, se hizo una prueba Anova multifactorial [22], para identificar las diferencias existentes entre las desviaciones estándar (Puntajes Z) en relación con las variables sociodemográficas.

Finalmente, para el relato de los hallazgos se triangularon los datos cualitativos y cuantitativos con las bases teóricas propuestas para el análisis. El software utilizado fue $\mathrm{IBM}^{\circledR}$ SPSS $^{\circledR}$ Statistic versión 23 (2017), versión licenciada de la Facultad Nacional de Salud Pública de la Universidad de Antioquia.

\section{Consideraciones éticas}

Para la investigación se tuvieron en cuenta el "Código de ética en investigación de la Universidad de Antioquia" [23] y la Resolución 008430 de 1993 del Ministerio de Salud [24]. El Comité de Ética que evaluó el proyecto lo clasificó como de riesgo mínimo (Acta 149 del 26 de agosto de 2016). 
Los participantes firmaron consentimiento informado, se garantizó el anonimato de la información y se desarrolló un evento municipal para socializar los resultados a los participantes y líderes comunitarios que participan en el proceso municipal de planeación local y presupuesto participativo (PLyPP) en Medellín.

Cabe señalar que el desarrollo de esta evaluación fue un proceso colaborativo y participativo entre el Estado (Secretaría de Salud de Medellín), la sociedad (ciudadanos de Medellín) y la academia (Facultad Nacional de Salud Pública de la Universidad de Antioquia).

\section{Resultados}

Este apartado se desarrolla teniendo en cuenta lo establecido en el modelo de teoría de cambio, que define el alcance de la intervención y los diferentes elementos interrelacionados; igualmente, se describen hallazgos de los momentos y procedimientos de socialización, convocatoria y selección de la población, además de los efectos que produjo la intervención en relación con la clasificación antropométrica de los niños y las niñas participantes según condiciones socioeconómicas, alimentarias y de salud, y la variación en los indicadores antropométricos $(\mathrm{P} / \mathrm{T})$ en niños y niñas menores de 5 años $\mathrm{e}$ IMC en mayores de 5 años. Finalmente, se describe unos cambios logrados no previstos, es decir, hallazgos que no habían sido tenidos en cuenta en el modelo de cambio, pero que fueron identificados durante la evaluación $y$, por ende, se reportan en este artículo.

\section{Delimitación del alcance de la evaluación mediante el modelo de teoría de cambio}

$\mathrm{Al}$ analizar los componentes de la intervención y su alcance durante la identificación del modelo de teoría del cambio, se encontró que solo se tenían definidos productos cuantitativos que obedecían a un número determinado de actividades, y no se tenían definidos los cambios específicos a lograr como resultado de la implementación de la intervención en la condición nutricional de los niños y las niñas. Además, la cantidad de actividades definidas sobrepasaba la capacidad operativa y de respuesta de los profesionales que implementaron la intervención. En síntesis, se planificó un alto número de actividades, con un problema explícito, pero sin la claridad del resultado plausible a lograr en un tiempo determinado, $\mathrm{y}$, con un limitado número de profesionales. Esto, a pesar de que los lineamientos técnicos de la intervención establecieran la necesidad de contar con un equipo interdisciplinario y con experiencia en el trabajo comunitario, como se requiere en los programas y proyectos que se implementan en la planeación del desarrollo local y del presupuesto participativo, dado su carácter comunitario y territorial [8].
El supuesto anterior de contar con el equipo interdisciplinario durante todas las actividades que contemplaba la intervención no se cumplió. Esto evidencia una implementación fragmentada, diferente a lo establecido en los protocolos de la intervención.

Otro supuesto necesario era la adherencia y aceptación de los niños y de sus cuidadores de las actividades de la intervención. Se observaron diferencias estadísticamente significativas y percepciones de clase social con respecto a los programas públicos. Al comparar por comuna, se observaron diferencias entre la comuna Castilla y El Poblado, con una mayor probabilidad de participación en tres o más actividades en el territorio de menor nivel socioeconómico (comuna de Castilla), comparado con el de mayor (comuna El Poblado) $(88,7$ y $16,7 \%$, respectivamente).

La siguiente cita da cuenta de las diversas actitudes de los padres frente a la intervención propuesta y de las dificultades inherentes a un proceso de sensibilización con padres o cuidadores cuando hay que superar prejuicios que pueden variar en virtud de sus diferencias socioculturales:

[...] hacer entender a los padres que era un programa para todos los niveles socioeconómicos fue difícil; algunos introyectan el mensaje, pero otros seguían insistiendo que era un programa para pobres [...] (emc14n171206_2). ${ }^{\dagger}$

La socialización, convocatoria y selección de la población: tensiones entre los actores

En la socialización se evidenciaron tensiones y relaciones de confrontación entre los líderes, el operador y la Secretaría de Salud, debidas a la disparidad entre lo priorizado por la comunidad según oferta institucional y lo presentado a implementarse por el operador, lo cual debilitó la participación de los líderes en los diferentes momentos de implementación del componente. Como señalaba un líder comunitario:

[...] vi el malestar de las comunidades, tanto de la comuna 5, Castilla, como de la comuna 14, El Poblado, porque ellos decían que lo que les habían ofertado (lo que habían votado) era una cosa totalmente diferente de lo que les venían a presentar [...] (emc5y14ie180226_7).

Las tensiones fueron agravadas con la participación de agentes primarios de salud contratados por el operador, pues, según los líderes de las comunas, los seleccionados por el operador no eran residentes de las comunas $y$, por ende, no contaban con experticia y conocimiento suficiente de los territorios. Las racionalidades generali-

$\dagger \quad$ La convención es la siguiente: Entrevista malnutrición comuna X [y Z], nutricionista (n), interventora externa (ie), abuela (a), niño (n), cuidadora (ca), fecha de realización_sacada de la línea Y del texto. 
zadas por los líderes confluyeron en que la Secretaría de Salud no quería que ellos participaran y, además, les impuso el proyecto y las personas que lo implementarían, como se evidencia en el siguiente relato:

[...] no fue fácil el trabajo de los agentes primarios de salud... las comunidades son... muy díscolas cuando personas extrañas... van a llegar a trabajar a estos sitios; entonces, prefieren que sean los líderes de cada una de las comunidades los que estén realizando esta labor (emc5y14ie180226_4).

Para la convocatoria y la selección de los beneficiarios hubo dificultades, puesto que los puntos de corte para clasificar los indicadores antropométricos basados en la Resolución 2465 del 2016 [21] eran muy estrechos; por ende, solo captaban niños y niñas con diagnóstico de sobrepeso y obesidad, y se dejaban de lado a quienes presentaban riesgo de sobrepeso, situación que retrasó de forma significativa el proceso de selección y la incor- poración de la totalidad de población de niños y niñas establecida para la intervención.

Clasificación antropométrica de los niños y niñas evaluados según condiciones socioeconómicas alimentarias y de salud

La comuna Castilla presentó el $83 \%$ de la población participante, y la comuna El Poblado, el 17 \%. En relación con las características socioeconómicas, el $13 \%$ pertenecen a familias desplazadas, $35 \%$ de las familias conformadas por mujeres cabezas de hogar. Madres y abuelos predominan como los cuidadores de los niños y las niñas, y entre el 85 y el $88 \%$ de las familias evaluadas reciben en promedio entre 1 y 2 salarios mínimos mensuales legales vigentes (SMMLV) (véase Tabla 1).

La siguiente cita da cuenta de la realidad que enfrentan las familias de niveles socioeconómicos bajos, encabezadas por mujeres que tiene a cargo un grupo familiar

Tabla 1. Distribución, de acuerdo con clasificación antropométrica, de los participantes de todas las actividades de la intervención "Malnutrición por exceso de peso en la primera infancia", 2017, según variables sociodemográficas.

\begin{tabular}{llcccccc}
\hline \multirow{2}{*}{ Variables sociodemográficas } & \multicolumn{2}{c}{ Sobrepeso } & \multicolumn{2}{c}{ Obesidad } & \multicolumn{2}{c}{ Total } \\
\cline { 2 - 7 } & No & Frecuencia & $\%$ & Frecuencia & $\%$ & Frecuencia & $\%$ \\
\hline \multirow{2}{*}{ Población desplazada } & Sí & 16 & 72,7 & 20 & 76,9 & 36 & 75 \\
& Sin dato & 3 & 13,7 & 3 & 11,6 & 6 & 13 \\
& No & 3 & 13,6 & 3 & 11,5 & 6 & 12 \\
Mujer cabeza de familia & Sín & 14 & 63,6 & 17 & 65,4 & 31 & 65 \\
& Abuela & 8 & 36,4 & 9 & 34,6 & 17 & 35 \\
Parentesco cuidador & Madre & 4 & 18,3 & 10 & 38,5 & 14 & 29 \\
& Padre & 16 & 72,7 & 11 & 42,3 & 27 & 56 \\
& Tío(a) & 1 & 4,5 & 2 & 7,7 & 3 & 6 \\
Ingresos promedio & Menos 1 smmLv & 1 & 4,5 & 3 & 11,5 & 4 & 9 \\
& Entre 1 y 2 smmLv & 17 & 87,5 & 25 & 96,2 & 42 & 88 \\
& Entre 3 y 4 smmLv & 5 & 10,4 & 0 & 0,0 & 5 & 10 \\
\hline
\end{tabular}

SMMLV: Salario mínimo mensual legal vigente.

extenso, donde la adquisición de alimentos se complejiza y, por lo tanto, optan por la compra de alimentos de baja calidad nutricional, con buen aporte de calorías:

La hija mía es muy guapa (valiente), trabaja en una casa de familia, se gana el mínimo. El papá de ninguno respondió. La plata no alcanza para muchas cosas, somos muchos. Lo que más compramos es salchichón, panes y panela, y hago aguapanela para todo el día $[\ldots]($ emc5a171126_1).

Al analizar las características relacionadas con la alimentación, se encontró que los cuidadores consideran el apetito como exagerado en los niños y las niñas clasificados como obesos; de igual forma, estos niños se caracterizan por comer entre 2 y 3 comidas al día y no consumen frutas.

Por otra parte, los cuidadores de niños y niñas clasificados con sobrepeso asumen el apetito como regular y se caracterizan por consumir de 4 a 5 comidas y de 1 a 2 porciones de frutas al día (véase Figura 2).

En relación con las condiciones de salud de los niños y las niñas, se identificó que el $23 \%$ presentó alguna enfermedad que comprometía el estado nutricional, donde predominaban las enfermedades respiratorias, con el $15 \%$, seguidas por las enfermedades 


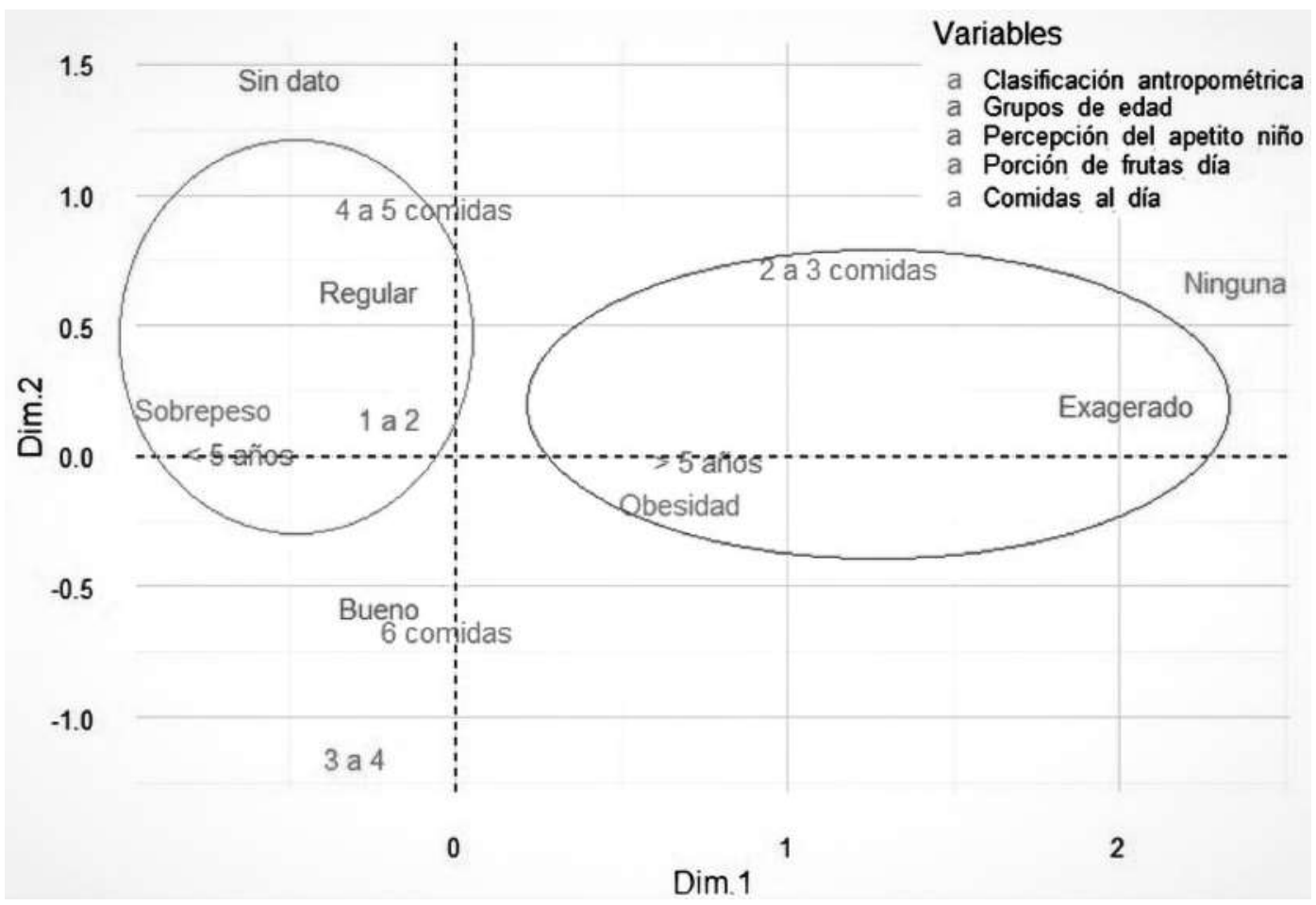

Figura 2. Análisis de correspondencia múltiple de la clasificación antropométrica (sobrepeso, obesidad) según variables alimentarias. Dim. 1 y Dim. 2 son las dimensiones en las que se reduce las variables analizadas, es decir, las cinco variables analizadas se resumen en dos dimensiones para explorar asociación.

Fuente: Equipo investigador U de A-FNSP, a partir de base de datos de información de ESE Metro Salud, 2017. Secretaría de Salud de Medellín.

infecciosas, con el $4 \%$, y las gastrointestinales y otras enfermedades, con el $2 \%$.

\section{Evaluación de los efectos}

Tabla 2. Comparación de muestras pareadas de acuerdo con el Puntaje $Z$ antes y después de la intervención nutricional en los niños y las niñas que participaron de todas las actividades, 2017.

\begin{tabular}{|c|c|c|c|c|c|c|c|}
\hline \multirow[b]{2}{*}{$\begin{array}{c}\text { Grupos de } \\
\text { edad }\end{array}$} & \multirow[b]{2}{*}{$\mathrm{N}$} & \multicolumn{2}{|c|}{ Iniciar tratamiento } & \multicolumn{2}{|c|}{ Final tratamiento } & \multirow[b]{2}{*}{ Diferencia mediana } & \multirow[b]{2}{*}{ Valor $\mathrm{p}$} \\
\hline & & Mediana & $\begin{array}{c}\text { Puntaje } \\
\text { Z }\end{array}$ & Mediana & $\begin{array}{c}\text { Puntaje } \\
\text { Z }\end{array}$ & & \\
\hline$<5$ años & 26 & 2,52 & 0,95 & 2,49 & 1,05 & $-0,03$ & 0,174 \\
\hline$\geq 5$ años & 22 & 2,4 & 0,83 & 2,3 & 0,64 & $-0,1$ & 0,236 \\
\hline Ambos grupos & 48 & 2,47 & 0,91 & 2,45 & 0,89 & 0,02 & 0,09 \\
\hline
\end{tabular}

Fuente: Equipo investigador Universidad de Antioquia-Facultad Nacional de Salud Pública (FNSP), a partir de base de datos de información de la

Empresa Social del Estado (ESE) Metro Salud, 2017. Secretaría de Salud de Medellín.
Al explorar los efectos de la intervención en los niños y las niñas según las variaciones del Puntaje Z del P/T para los niños y las niñas menores de 5 años, y el IMC/ TALLA para los de 5 o más años, antes y después de la intervención se observaron cambios en estos indicadores, beneficiosos para la población (véase Tabla 2). 
Para determinar si las variables sociodemográficas (sexo, estrato socioeconómico, etnia, régimen en salud, grupos de edad y población desplazada) y la combinación de sus interacciones podrían tener influencias sobre las variaciones del Puntaje Z, se desarrolló un modelo de Anova multifactorial y se obtuvo como resultado que las variables sociodemográficas no influyen sobre las variaciones en el Puntaje Z; sin embargo, la interacción presentada entre las variables de estrato socioeconómico y grupos de edad podría influenciar las variaciones del Puntaje Z (véase Tabla 3). Además, los datos cualitativos aportados por las niñas y los niños dan cuenta de algunos cambios en hábitos nutricionales y estilos de vida que pueden mostrar esa variación en el IMC.

Tabla 3. Anova multifactorial de las diferencias en las variaciones del Puntaje $Z$ en los niños y las niñas que participaron en todas las actividades desarrolladas en el componente, de acuerdo con las variables sociodemográficas y sus interacciones.

\begin{tabular}{lccccc}
\hline \multicolumn{1}{c}{ Factores } & Suma de cuadrados & gl & Cuadrático promedio & F & p-valor \\
\hline Modelo corregido & $4,748^{a}$ & 22 & 0,22 & 1,52 & 0,16 \\
Interceptación $^{b}$ & 0,16 & 1 & 0,16 & 1,12 & 0,3 \\
Sexo & 0,38 & 1 & 0,38 & 2,66 & 0,12 \\
Estrato & 0,08 & 3 & 0,03 & 0,18 & 0,91 \\
Etnia & 0 & 0 & $d$ & $d$ & d \\
Régimen en salud & 0,38 & 2 & 0,19 & 1,34 & 0,28 \\
Grupos de edad & 0 & 1 & 0 & 0,02 & 0,88 \\
Población desplazada & 0,42 & 2 & 0,21 & 1,48 & 0,25 \\
Estrato grupos de edad $^{c}$ & 1,3 & 1 & 1,3 & 9,17 & $0,01^{c}$ \\
\hline
\end{tabular}

gl: Los grados de libertad son la cantidad de información en los datos.

$\mathrm{F}$ : Un valor $\mathrm{F}$ suficientemente grande indica que el modelo es significativo.

a. $\mathrm{R}^{2}=0,572\left(\mathrm{R}^{2}\right.$ ajustada $\left.=0,196\right)$.

b. La interceptación es la combinación entre las variables a analizar; se busca observar la asociación entre ellas.

c. La interacción entre esas variables es significativa.

d. No existe una mínima asociación en la interacción de las variables.

Fuente: Equipo investigador Universidad de Antioquia-FNSP, a partir de base de datos de información de ESE Metro Salud, 2017. Secretaría de Salud de Medellín.

En el siguiente testimonio se muestra cómo un niño que estuvo expuesto a la intervención da cuenta de los cambios de sus hábitos de salud y prácticas alimentarias:

[...] A mí ya no me gusta la gacheocha Coca-Cola. El médico me dijo que no la podía tomar, pero ver televichor sí me gusta mucho, pero ya veo más potito; también jugar en el parque [...] (emc5n171113_2).

\section{Cambios no previstos por la intervención}

Uno de los cambios no previstos fueron las variaciones en el índice de masa corporal de las madres y cuidadores de los niños y las niñas. Este dato se obtuvo al evaluar los cuidadores participantes, quienes contaban con las mediciones del índice de masa corporal al inicio y al final de la intervención (véase Tabla 4). En estos cuidadores se encontró que quienes fueron clasificados con obesidad y sobrepeso se caracterizaban por residir en el estrato 2, y los clasificados con obesidad mórbida se caracterizaron por ser de estrato 3 y tener ingresos promedio mensuales de 1 a 2 SMMLV.

En relación con los hábitos alimenticios, los cuidadores clasificados con obesidad y obesidad mórbida se caracterizaron por consumir al día de 2 a 3 comidas, 3 a 4 bebidas azucaradas y no consumir agua, entre quienes al finalizar la intervención no mejoró el estado nutricional. Por otra parte, los cuidadores clasificados con peso adecuado se caracterizaron por consumir al día de 1 a 2 porciones de frutas, de 1 a 2 bebidas azucaradas y consumir agua durante el día, y mejoró su estado nutricional al finalizar la intervención. Por su parte, los cuidadores clasificados con sobrepeso se caracterizaron por consumir al día de 4 a 5 comidas. De este modo, los datos cualitativos corroboran los datos cuantitativos. 
Tabla 4. Comparación de muestras pareadas de acuerdo con el índice de masa corporal antes y después de la intervención nutricional en los cuidadores de las comunas Castilla y El Poblado, año 2017.

\begin{tabular}{|c|c|c|c|c|c|c|c|}
\hline \multirow[b]{2}{*}{ Población } & \multirow[b]{2}{*}{$\mathrm{N}$} & \multicolumn{2}{|c|}{ Iniciar tratamiento } & \multicolumn{2}{|c|}{ Final tratamiento } & \multirow[b]{2}{*}{ Diferencia media } & \multirow[b]{2}{*}{ Valor $\mathrm{p}$} \\
\hline & & Media & $\begin{array}{c}\text { Puntaje } \\
\text { Z }\end{array}$ & Media & $\begin{array}{c}\text { Puntaje } \\
\text { Z }\end{array}$ & & \\
\hline Cuidadores & 33 & 30,91 & 7,07 & 30,71 & 6,99 & 0,2 & 0,042 \\
\hline
\end{tabular}

Fuente: Equipo investigador Universidad de Antioquia-FNSP, a partir de base de datos de información de ESE Metro Salud, 2017. Secretaría de Salud de Medellín.

El siguiente testimonio refleja los hábitos de consumo alimentario que llevaban a cabo los cuidadores clasificados con obesidad mórbida y quienes después de la intervención no mejoraron su estado nutricional

Yo a veces sólo me como el desayuno y ya espero hasta por la noche; pero sí tomo mucho aguapanela. El agua sola no me gusta y las frutas muy poquito. Me fascinan los juguitos de cajita [...] (emc14ca171106_4).

\section{Discusión}

La variación positiva de los puntajes $\mathrm{Z}$, reflejada en la mejoría de la clasificación nutricional de algunos niños, se explica por el compromiso, la aceptabilidad y la adopción de las recomendaciones nutricionales y psicosociales por parte de los padres o cuidadores, que, sin lugar a duda, se enmarcaron en una participación de cooperación y de tipología sinérgica durante la intervención, en la que confluyeron la participación positiva y las relaciones empáticas fortalecidas por los profesionales, los cuales demostraron estar bien cualificados para el manejo integral de la obesidad infantil.

Se ha identificado la relación de las condiciones económicas y sociales y la obesidad, y su interacción con factores biológicos, sociales y culturales [25]. En esta evaluación, reportamos que esta relación es directa, ya que los datos cualitativos y los cuantitativos evidencian condiciones socioeconómicas poco favorables que benefician ambientes obesogénicos, emergiendo el fenómeno de obesidad enmascarada en la pobreza.

Frente a dicho fenómeno, un estudio denominado "La obesidad en la pobreza: un nuevo reto para la salud pública" expresa la modificación de conductas alimentarias en contextos urbanos con alto consumo de grasas y azúcar, y limitado consumo de fibra. Además, aparecen los precios de las frutas y verduras como una barrera para su consumo, y una focalización de la industria alimenticia en estos grupos poblacionales con alimentos altos en grasas, azúcares y poco nutritivos [26].

A lo anterior se suman representaciones culturales acerca del sobrepeso y la obesidad, sustentadas en que un niño gordito es más bonito y saludable, como lo mostraron los resultados cualitativos, lo que reitera la dis- torsión de la imagen corporal que perciben los padres o cuidadores, incluyendo las abuelas, frente al estado nutricional de sus hijos o nietos. Se evidencia, además, que existe un desconocimiento de las consecuencias para la salud que el sobrepeso y la obesidad acarrean en las subsecuentes etapas de la vida. Esto se ratifica con los aportes que hace la evidencia científica [27].

Si se consideran los diferentes factores que convergen para intervenir de forma integral el sobrepeso y la obesidad infantil y, en vista de que la evaluación develó que no se cumplió con una de las supuestos - que era contar con el equipo interdisciplinario durante todo el momento de la intervención-, la evidencia científica es clara y sugiere que la efectividad en este tipo de intervenciones requiere un equipo interdisciplinario compuesto de pediatras, enfermeras, médicos de familia, dietistas, psicólogos y psiquiatras durante un tiempo aproximado de un año de intervención, a fin de lograr la transdisciplinariedad, mediante acciones de educación para la salud, en el marco de la atención primaria en salud, que permita el logro de los objetivos [28].

Por otro lado, uno de los cambios no previstos evidenciados en la evaluación fueron cambios estadísticamente significativos en la variación del IMC de los cuidadores de los niños y las niñas. Esto se logró evidenciar tanto desde los análisis cuantitativos como de los cualitativos. Hallazgos similares se reportan en otro estudio, cuyo modelo de intervención presenta características similares a esta intervención, donde se identificaron cambios relacionados con el manejo de porciones, adopción de una dieta pobre en grasas y sustitución de frituras por vegetales [29]. Esto, considerando la relación existente de la obesidad infantil con los antecedentes de los padres [30].

Otro de los elementos a destacar son los factores que intervienen en la adherencia a cualquier programa de salud pública, haciendo visible la importancia de realizar lecturas de contexto, entender las particularidades de cada territorio, las formas como se relacionan los actores y cómo perciben su proceso de salud-enfermedadrehabilitación [31].

Es imprescindible fortalecer la cultura evaluativa de los procesos de planificación e implementación de las polí- 
ticas y los programas en salud pública, con el fin de obtener evidencia científica, mejorar las intervenciones y retomar las recomendaciones brindadas para la construcción o mejoras de los componentes, en términos de disponibilidad de recursos humanos, físicos y adaptación de la experiencia según los determinantes sociales de los territorios.

Una de las limitaciones del estudio fue no iniciar paralelamente la evaluación al momento de comenzar la intervención, dada la diferencia en los tiempos administrativos y contractuales de ambos proyectos, asuntos que deben considerarse desde la formulación de los proyectos, comprendiendo que la evaluación hace parte integral de los mismos. Esta situación implicó que el equipo de investigación no estuviera desde el inicio de la intervención en algunas actividades propias de la implementación, por lo que se acudió a otras fuentes para obtener información suficiente y de calidad.

Es importante señalar que la intervención de sobrepeso y obesidad se convirtió en un espacio de tensiones, confrontaciones y racionalidades, que desfavorecen la participación, y posicionaron nuevamente prácticas de democracia representativa y no participativa. Por ende, se recomienda que la lógica institucional sea clara y convincente en sus explicaciones frente a la oferta institucional que presenta, pues la falta de comunicación da motivos a tensiones, lo que hace surgir dudas sobre el proceso y relega a la comunidad a ser mera observadora y receptora de políticas públicas.

Finalmente, el modelo de teoría del cambio se constituye en una adecuada herramienta a utilizar en la evaluación de programas y proyectos de salud pública y nutrición poblacional. En nuestro caso, permitió redefinir los alcances de la intervención "Malnutrición por exceso de peso en niños y niñas" de manera constructiva con los actores. Igualmente, la evaluación de los efectos mostró que las acciones programáticas de la intervención favorecieron el alcance del cambio global estipulado, es decir, de las variaciones del IMC y, por consiguiente, permitieron mejoras en el estado nutricional y de salud, no solo de los niños y las niñas, sino también de las madres o cuidadores, en la medida en que estos apropiaron algunos hábitos y estilos de vida saludables. Esto demuestra que el programa y sus intervenciones pueden replicarse a partir de algunos ajustes, y dándole mayor protagonismo a los procesos que posibilitaron dichos cambios, sin dejar de lado el marco institucional y de política pública que rige el accionar democrático de la PLyPP.

\section{Agradecimientos}

A los participantes del estudio (a los niños, las niñas y sus padres, y a los profesionales de la intervención); al equipo general de esta investigación, perteneciente a la Facultad Nacional de Salud Pública de la Universidad de Antioquia por su compromiso, disposición y paciencia. A los delegados y las delegadas de las comisiones de salud del Presupuesto Participativo de Medellín y al equipo territorial de la Dirección Técnica de Planeación, de la Secretaría de Salud de Medellín, por sus aportes. Finalmente, al equipo y la jefatura del Centro de Extensión de la Facultad Nacional de Salud Pública, por su compromiso y gestión durante el desarrollo de este proyecto. A los evaluadores de la revista y su equipo editorial, por sus acertados comentarios para la mejora de este manuscrito. Al profesor Jaime Andrés Gaviria por su asesoría estadística.

\section{Declaración de financiación}

Convenio 4600071225 de 2017, entre la Alcaldía de Medellín y la Universidad de Antioquia.

\section{Declaración de conflicto de intereses}

Los autores declaramos que no tenemos ningún tipo de conflicto de intereses relacionado con el presente artículo.

\section{Declaración de responsabilidad}

Los autores declaran que los puntos de vista expresados son responsabilidad personal y no de la institución de afiliación ni de la institución de financiación.

\section{Declaración de autoría}

- Cristina María Mejía Merino. Aportó a la concepción de la idea original del trabajo. Analizó e interpretó los datos cualitativos y cuantitativos, participó en la redacción del manuscrito y en la aprobación final de la versión a publicar.

- Camilo Noreña Herrera. Desarrolló la idea original del trabajo, participó en la redacción del manuscrito y en la aprobación final de la versión a publicar.

- Santiago Flórez Vanegas. Analizó e interpretó los datos cuantitativos, participó en la redacción del manuscrito y en la aprobación final de la versión a publicar.

- Alejandra Marín Uribe. Participó en el análisis e interpretación de los datos cuantitativos y cualitativos, participó en la redacción del manuscrito y en la aprobación final de la versión a publicar.

- Verónica María Lopera Velásquez. Desarrolló la idea original del trabajo, participó en la redacción del manuscrito y en la aprobación final de la versión a publicar.

\section{Referencias}

1. Centro de prensa Organización Mundial de la Salud. La obesidad entre los niños y los adolescentes se ha multiplicado por 10 en los cuatro últimos decenios [internet]; 2017 [citado 2020 jun. 15]. Disponible en: http://www.who.int/es/news-room/detail/11- 
10-2017-tenfold-increase-in-childhood-and-adolescent-obesityin-four-decades-new-study-by-imperial-college-london-and-who

2. Urrutia Alarcón LF. Análisis del referencial de las políticas públicas sobre prevención de la obesidad infantil en Colombia [Trabajo final de maestría]. [Bogotá]: Universidad Nacional de Colombia; 2017 [citado 2019 nov. 3]. Disponible en: http://bdigital.unal.edu. co/57509/7/LuisFe.Alarc\%C3\%B3nUrrutia.2017.pdf

3. Hurtado C, Mejía C, Mejía F, et al. Malnutrición por exceso y déficit en niños, niñas y adolescentes, Antioquia, 2015. Rev. Fac. Nac. Salud Pública. 2017;35(1):58-70. Dor: https://doi.org/10.17533/ udea.rfnsp.v35n1a07

4. Alcaldía del Municipio de Medellín. Profundización del análisis de la situación de salud; Medellín 2005-2015; 2017.

5. Alcaldía de Medellín. Plan de seguridad alimentaria y nutricional del municipio de Medellín 2016-2028. Medellin; 2015.

6. Gómez L, Miguel S. Programa de Educación para la salud: prevención de la obesidad en niños de entre 11 y 14 años de Sant Andreu de la Barca [Tesis de grado]: Escola Universitària d'Infermeria Gimbernat, Sant Cugat del Vallès; 2015. [citado 2019 nov. 3] Disponible en: http://eugdspace.eug.es/xmlui/bitstream/handle/123456789/372/Prevenci\%C3\%B3n\%20de\%201a\%20obesidad $\% 20$ en $\% 20$ ni $\%$ C3\%B1 os $\% 20 \mathrm{de} \% 20$ entre $\% 2011 \% 20 \mathrm{y} \% 20$ $14 \% 20 \mathrm{a} \% \mathrm{C} 3 \% \mathrm{~B} 1$ os $\% 20 \mathrm{de} \% 20$ Sant $\% 20$ Andreu $\% 20 \mathrm{de} \% 20$ la $\% 20$ Barca.pdf?sequence $=1 \&$ isAllowed $=y$

7. Perea A, López GE, Padrón M, et al. Evaluación, diagnóstico, tratamiento y oportunidades de prevención de la obesidad. Acta Pediátr Méx. 2014;35(4):316-37.

8. Concejo de Medellín. Acuerdo Municipal 43 de 2007, por el cual se crea e institucionaliza la Planeación Local y el Presupuesto Participativo en el marco del Sistema Municipal de Planeación -Acuerdo 043 de 1996 - y se modifican algunos de sus artículos (2007 noviembre 8).

9. Rodríguez NS. Tendencias actuales en la evaluación de políticas públicas. Ens. Econ. [internet]. 2018 [citado 2020 jun. 15]; 28(53):15-35. Dor: http://dx.doi.org/10.15446/ede.v28n53.75382

10. Castro VE. Evaluación de políticas públicas: contexto, tendencias y desafíos para la realidad argentina. Rev IISE [internet]. 2014 [citado 2020 jun. 15]; 6(6):19-34. Disponible en: http://www.ojs. unsj.edu.ar/index.php/reviise/article/view/54/53

11. Ortiz A, Rivero G. Desmitificando la teoría del cambio. México: Building Capacity Wordwide Pact [internet]; 2007 [citado 2020 jun. 15]. Disponible en: https://planificacionsocialunsj.files.wordpress.com/2011/09/demistificando-la-teoria-del-cambio.pdf

12. Cardozo Brum M. Políticas públicas: los debates de su análisis y evaluación. Andamios [internet]. 2013 [citado 2020 jun. 15]; 10(21):39-59. Disponible en: http://www.scielo.org.mx/scielo. php?script $=$ sci_arttext\&pid $=$ S1870-

13. Peters D, Tran NT, Adam T. Investigación sobre la implementación de políticas de salud: guía práctica. Ginebra: World Health Organization [internet]; 2013 [citado 2019 nov. 3]. Disponible en: https://www.who.int/alliance-hpsr/resources/Implementation Research_SP.pdf?ua $=1$

14. Ridde V, Dagenais C, compiladores. Enfoques y prácticas en la evaluación de programas. Bogotá: Pontificia Universidad Javeriana [internet]; 2015 [citado 2019 nov. 3]. Disponible en: https:// www.researchgate.net/publication/295869551_Enfoques_y_ practicas en la evaluacion de programas
15. Retolaza Eguren I. Teoría de cambio: un enfoque de pensamiento-acción para navegar en la complejidad de los procesos de cambio social. PNUD [internet]; 2010 [citado 2020 jun. 15]. Disponible en: https://xarxanet.org/sites/default/files/pnud-hivos-guia_teoria_de_cambio.pdf

16. Patton MQ. Utilization-Focused Evaluation. Los Angeles- London-New Dehli- Singapore: Sage; 2008.

17. Patton M-Q. La evaluación centrada en la utilización. En: Enfoques y prácticas en la evaluación de programas. Bogotá: Pontificia Universidad Javeriana; 2015.

18. Pluye P, Potvin L, Denis JL. Making public health programs last: Conceptualizing sustainability. Eval Program Plann. 2004;27(2):12133. DoI: https://doi.org/10.1016/j.evalprogplan.2004.01.001

19. Jara MI. Enfoque y prácticas en la evaluación de programas [Reseña]. Rev. Gerenc. Polit. Salud. [internet] 2016 [citado 2019 nov. 3]; 15(30): 251-252. http://www.scielo.org.co/scielo. php?script $=$ sci_arttext\&pid=S1657-70272016000100017

20. Galeano ME. Intervención de investigación social cualitativa: el giro de la mirada. Medellín: Fondo Editorial FCSH; 2018.

21. Colombia, Ministerio de Salud y Protección Social. Resolución 2465 , por la cual se adoptan los indicadores antropométricos, patrones de referencia y puntos de corte para la clasificación antropométrica del estado nutricional de niñas, niños y adolescentes menores de 18 años de edad, adultos de 18 a 64 años de edad y gestantes adultas y se dictan otras disposiciones (2016 junio 14).

22. Martínez M, Sánchez A, et al. Bioestadística amigable. 3. ${ }^{\mathrm{a}}$ ed. Barcelona: Elsevier; 2014.

23. Universidad de Antioquia. Código de ética en investigación de la Universidad de Antioquia [internet]. s. f. [citado 2020 sep. 25]. Disponible en: http://www.udea.edu.co/wps/wcm/connect/udea/ e79da6b4-1402-496b-88bc-0dc0321ba827/codigo-etica-udea. pdf?MOD=AJPERES

24. Colombia, Ministerio de Salud. Resolución 008430, por la cual se establecen las normas científicas, técnicas y administrativas para la investigación en salud (1993 oct. 4).

25. Figueroa D. Obesidad y pobreza : marco conceptual para su análisis en Latinoamérica. Saúde Soc São Paulo. 2009(1);18:103-17.

26. Peña $M$, Bacallao Jorge. La obesidad en la pobreza: un nuevo reto para la salud pública. Washinton: Organización Panamericana de la Salud; 2000.

27. Romero IM, Abdala C, Salas V. Informe final de evaluación. Programa de tratamiento de la obesidad: Alimentación saludable y actividad física (en adultos, niños y adolescentes) y obesidad mórbida; Chile: Ministerio de Salud; 2008.

28. Flores ME, Elías AR. Intervenciones dirigidas a disminuir/evitar el sedentarismo en los escolares. Enferm. Univ. [internet]. 2012 [citado 2019 nov. 3]; 9(4):45-56.

29. Pedraz CG. "La mujer ha tenido un papel fundamental en el desarrollo de la ciencia de la Nutrición". Agencia Iberoamericana para la difusión de la ciencia y la tecnología [internet]; 2019 [citado 2019 nov. 3]. Disponible en: http://www.dicyt.com/viewNews. php?newsId $=27407$

30. Villagrán S, Rodríguez A, Novalbos PJ, et al. Hábitos y estilos de vida modificables en niños con sobrepeso y obesidad. Nutr Hosp. [internet]. 2010 [citado 2019 nov. 3]; 25(5):823-31.

31. Martínez M, García I, Estrada B. Adherence to nutritional therapy: Intervention based on motivational interviewing and brief solution-focused therapy. Rev Mex Trastor Aliment. 2016;7(1):3239. DoI: https://doi.org/10.22201/fesi.20071523e.2016.1.353 